\title{
Broad Band THz Sensing by 2DEG Bow-Tie-Type Diodes
}

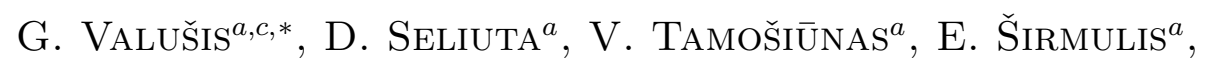
S. BAlAkAuskas ${ }^{a}$, J. GradAuskas $^{a}$, A. SuŽIEdĖlis $^{a}$, S. Ašmontas ${ }^{a}$, T. Anbinderis ${ }^{b}$, A. NArkunnas $^{b}$, I. PApsujeva $^{b}$, A. Lisauskas ${ }^{c}$, H.G. Roskos $^{c}$ AND K. KÖHLER ${ }^{d}$

${ }^{a}$ Semiconductor Physics Institute, A. Goštauto 11, 01108 Vilnius, Lithuania

${ }^{b}$ Elmika Ltd, Naugarduko 41, 03227 Vilnius, Lithuania

${ }^{c}$ Physikalisches Institut der J.W. Goethe-Universität 60054 Frankfurt/M, Germany

${ }^{d}$ Fraunhofer-Institut für Angewandte Festkörperphysik 79108 Freiburg, Germany

We suggest a novel approach to detect broad band, 0.078-2.52 THz, electromagnetic radiation at room temperature using an asymmetrically-shaped bow-tie diode based on a modulation-doped GaAs/AlGaAs structure. We show that the voltage sensitivity in the range from $0.078 \mathrm{THz}$ up to $0.8 \mathrm{THz}$ has a plateau and its value is within $0.3-0.5 \mathrm{~V} / \mathrm{W}$. We consider the bow-tie diode design to increase the sensitivity of the device.

PACS numbers: 73.50.- - h, 85.30.De, 72.30.+q

\section{Introduction}

Electromagnetic radiation of terahertz/subterahertz (THz/subTHz) range is becoming increasingly important in a large variety of scientific and practical applications, for instance, in detecting molecules in the galactic clouds, DNA profiling, biomedical imaging as well as identifying chemical signatures or in guiding a missile to target. For a widespread adoption and commercialization of $\mathrm{THz}$ electronics the essential task is to develop compact, low input power solid-state $\mathrm{THz}$ sources and reliable detectors.

Two-dimensional electron gas (2DEG) is an essential ingredient in many modern THz sensors. For instance, 2DEG embedded in a gated field-effect transistor can be used to detect the $\mathrm{THz}$ radiation in both, selective and non-resonant

*corresponding author; e-mail: valusis@pfi.lt 
way $[1,2]$. The structure with a 2DEG channel integrated in a log-periodic antenna and placed in a strong magnetic field at liquid helium temperatures can serve as a high-sensitivity quantum Hall photodetector [3].

In this communication, we present a novel approach to detect broad band $\mathrm{THz}$ radiation at room temperature. We investigate an asymmetrically-shaped bow-tie diode based on a modulation-doped GaAs/AlGaAs structure and show its operation characteristics within $0.078-2.52 \mathrm{THz}$ range. We discuss both experimental and theoretical ways to optimize the design of the suggested bow-tie diodes, aiming to increase their voltage sensitivity.

\section{Design of bow-tie diodes and experimental techniques}

The shape of the bow-tie diode and its structure are given in Fig. 1. It is seen that the left leaf of the bow-tie is metallized (black colour) in order to concentrate the incident radiation into the apex of the right leaf which contains the 2DEG layer. The electrons are heated in the layer non-uniformly by incident $\mathrm{THz}$ radiation causing a voltage signal over the ends of the diode. Therefore, no bias voltage is required for the diode operation. On the other hand, the asymmetric bow-tie shape, apart from detection, also acts as a coupler of a broad band $\mathrm{THz}$ radiation.

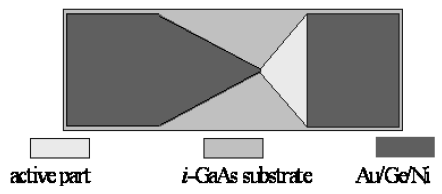

Fig. 1. Planar shape of the device. It resembles bow-tie antenna and acts simultaneously as a coupler of a $\mathrm{THz}$ radiation. The diode was processed into a $2 \mu \mathrm{m}$ depth mesa. The length and width of the device is $500 \mu \mathrm{m} \times 100 \mu \mathrm{m}$, whilst the size of the apex is $12 \mu \mathrm{m}$. The length of active part amounts to $50 \mu \mathrm{m}$. The perpendicular structure of the active part (layer sequence from the top of the diode): $20 \mathrm{~nm}$ i-GaAs cap layer; $80 \mathrm{~nm}$ Si-doped, $10^{18} \mathrm{~cm}^{-3}$, layer of $\mathrm{Al}_{0.25} \mathrm{Ga}_{0.75} \mathrm{As}$, undoped spacer, $45 \mathrm{~nm} \mathrm{Al}_{0.25} \mathrm{Ga}_{0.75} \mathrm{As}$, $1000 \mathrm{~nm}$ i-GaAs; twenty periods of $9 \mathrm{~nm} \mathrm{Al}_{0.25} \mathrm{Ga}_{0.75} \mathrm{As} / 1.5 \mathrm{~nm}$ GaAs layers; $0.5 \mu \mathrm{m}$ i-GaAs; semi-insulating substrate.

The devices were studied in two THz radiation ranges, $0.078-0.118 \mathrm{THz}$ and 0.584-2.52 THz. As the emission sources, we have used a backward wave oscillator and an optically-pumped molecular $\mathrm{THz}$ laser. In experiments the electric field of the incident $\mathrm{THz}$ radiation $E_{z}$ was oriented along the sample.

\section{Experimental results and discussion}

As already mentioned, the principle of the device operation is based on non-uniform charge carrier heating in the 2DEG layer. The non-uniform heating can be nicely illustrated by modeling the distribution of the electric field amplitude in 
the diode (Fig. 2) calculated by a three-dimensional finite-difference time-domain method. The modeling was performed at $0.75 \mathrm{THz}$ for two types of diodes having different sizes of the apex, $12 \mu \mathrm{m}$ and $3 \mu \mathrm{m}$. From Fig. 2 one can see that the electric field concentrates in the vicinity of the apex, meanwhile, its amplitude decreases rapidly over $10 \mu \mathrm{m}$ range. This trend was found for both types of the diodes. Furthermore, it is worth noting that the narrower apex is the larger the amplitude of the electric field is reached. In $12 \mu \mathrm{m}$ apex diode, the amplitude of the field in the apex is larger by factor of 3 than the field amplitude 20-25 $\mu \mathrm{m}$ away, while for $3 \mu \mathrm{m}$ apex, the corresponding factor is close to 5 . Therefore, the calculations indicate that the reduction of the apex size is one of the ways to increase the voltage sensitivity of the device.

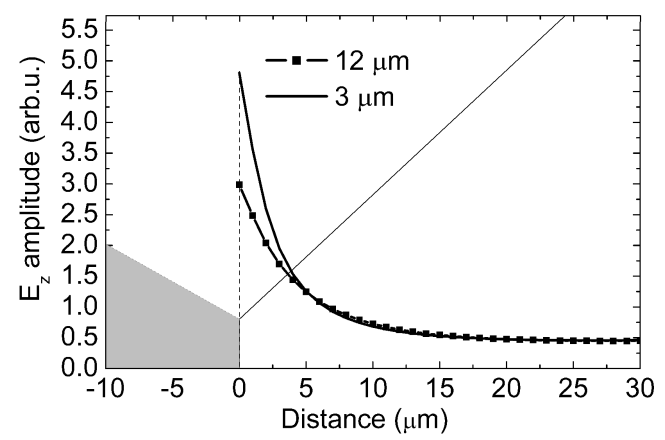

Fig. 2. Distribution of the amplitude of the $\mathrm{THz}$ electric field in the active part of the bow-tie diode exposed to radiation of $0.75 \mathrm{THz}$ frequency. Calculations were made using three-dimensional finite-difference time-domain method. Distance is measured from the apex. The background of the insert is decorated schematically as the shape of the device.

This idea was tested within a wide range of $\mathrm{THz}$ frequencies. Figure 3 presents results of the voltage sensitivity as a function of frequency for the diodes with $12 \mu \mathrm{m}$ and $3 \mu \mathrm{m}$ apexes. Circles and diamonds denote the experimental data while the solid line is fit made using the phenomenological approach of Ref. [4]. It is seen that the frequency dependence of the voltage sensitivity has two characteristic ranges: the first one is from $0.078 \mathrm{THz}$ up to $0.8 \mathrm{THz}$ - here the sensitivity is nearly independent of frequency and is close to $0.3 \mathrm{~V} / \mathrm{W}$ for the device with $12 \mu \mathrm{m}$ apex and about $0.5 \mathrm{~V} / \mathrm{W}$ for device with $3 \mu \mathrm{m}$ apex. One must note that the relative increase in the signal agrees well with the theoretical predictions on electric field increase in the apex given in Fig. 2. In the second frequency rangeabove $0.8 \mathrm{THz}$ - the experimental points of the sensitivity drop more abruptly than the phenomenological approach predicts. We explain the more pronounced decrease in the sensitivity by somewhat weaker coupling of the $\mathrm{THz}$ radiation at higher frequencies. As in the lower frequency range, the narrower apex device is found to be more sensitive. Thus, we suppose that such an approach can be successfully used to improve the device operation. 


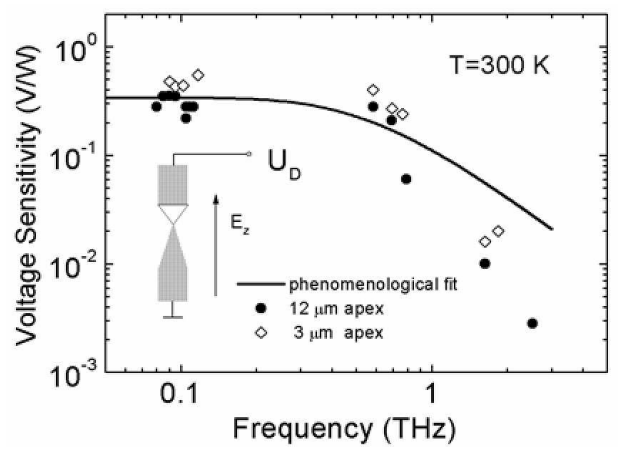

Fig. 3. Voltage sensitivity as a function of frequency for the bow-tie 2DEG diodes having different sizes of the apex. Circles and diamonds denote experimental data, solid line shows fit using phenomenological approach [4]. Inset depicts schematic view of the measurement scheme.

An alternative way to increase the sensitivity is to employ high mobility of the 2DEG at low temperatures since the carrier heating is proportional to this parameter. We demonstrated the effect of electron mobility very recently in Ref. [5]: it was determined that the sensitivity increases proportionally to the electron mobility, and the values of the sensitivity for the diode with $12 \mu \mathrm{m}$ apex and having the same design as given here is about $20 \mathrm{~V} / \mathrm{W}$.

\section{Acknowledgments}

The work was partly supported by NATO SfP project 978030 "Broad Band Detectors" and Lithuanian State Science and Studies Foundation under contract V-04073. A.S. acknowledges EC for the possibility to prepare diodes with $3 \mu \mathrm{m}$ apex at the Braun Submicron Research Center (contract No. HPRI-CT-200100114). G.V. is grateful to the Alexander von Humboldt Foundation for supporting the research at the Frankfurt/M University labs.

\section{References}

[1] W. Knap, Y. Deng, S. Rumyantsev, M.S. Shur, Appl. Phys. Lett. 81, 4637 (2002).

[2] W. Knap, V. Kachorovskii, Y. Deng, S. Rumyantsev, J.-Q. Lü, R. Gaška, M. Shur, G. Simin, X. Hu, M. Asif Khan, C.A. Saylor, L.C. Brunel, J. Appl. Phys. 91, 9346 (2002).

[3] Y. Kawaguchi, K. Hirakawa, S. Komiyama, Appl. Phys. Lett. 80, 3418 (2002).

[4] S. Ašmontas, A. Sužiedèlis, Int. J. Infrared Millim. Waves 15, 525 (1994).

[5] D. Seliuta, V. Tamošiūnas, E. Širmulis, S. Ašmontas, A. Sužiedèlis, J. Gradauskas, G. Valušis, P. Steenson, W.-H. Chow, P. Harrison, A. Lisauskas, H.G. Roskos, K. Köhler, Proc. 27th Int. Conf. on Physics of Semiconductors, Flagstaff, Arizona, 26-30 July 2004, submitted for publication. 